\title{
Clinical application value of brain CT perfusion imaging in the treatment of acute ischemic stroke thrombolytic therapy
}

\author{
SHIMING XU, LI WANG and LIDONG ZHAO
}

Department of Imaging, Liaocheng Brain Hospital, Liaocheng, Shandong 252000, P.R. China

Received November 13, 2018; Accepted March 1, 2019

DOI: 10.3892/etm.2019.7431

\begin{abstract}
Clinical application value of CT perfusion (CTP) imaging in the treatment of acute ischemic stroke (AIS) thrombolytic therapy was investigated. Retrospective analysis of 185 suspected AIS patients in Liaocheng Brain Hospital from April 2012 to December 2017 were involved. We observed the non-contrast CT (NCCT) and CTP in the diagnosis of AIS patients, and recorded as well as compared CTP parameters of AIS patients. The National Institute of Health Stroke Scale (NIHSS) rating was conducted, and the correlation between the prognosis of NIHSS scores and CTP parameters was investigated. A total of 177 patients were diagnosed with AIS. The sensitivity levels of CTP examination for diagnosis of AIS patients was significantly higher than that of the NCCT examination $(\mathrm{P}<0.050)$. The diagnostic compliance rates of CTP examination was significantly higher than that of the NCCT examination $(\mathrm{P}<0.050)$. The cerebral blood volume $(\mathrm{CBV})$ in the abnormal perfusion area was significantly lower than that in the mirror side zone $(\mathrm{t}=21.160, \mathrm{P}<0.001)$. The prognostic NIHSS score was negatively correlated with CBV in patients with thrombolytic therapy $(r=-0.912, \mathrm{P}<0.001)$. The cerebral blood flow $(\mathrm{CBF})$ in the abnormal perfusion zone was significantly lower than that in the mirror side zone $(t=19.170$, $\mathrm{P}<0.001)$. The prognosis of patients with thrombolytic therapy was negatively correlated with $\mathrm{CBF}(\mathrm{r}=-0.915, \mathrm{P}<0.001)$. The mean transit time (MTT) in the abnormal perfusion zone was higher than that in the mirror side zone $(\mathrm{t}=13.480, \mathrm{P}<0.001)$. NIHSS scores were obtained 3 months after thrombolytic therapy and found that the prognostic NIHSS scores were positively correlated with MTT $(\mathrm{r}=0.887, \mathrm{P}<0.001)$. The results indicated that brain CTP has a high diagnostic value for intravenous thrombolytic therapy in AIS and there was a significant correlation between the prognosis scores of patients. Thus, it is
\end{abstract}

Correspondence to: Dr Lidong Zhao, Department of Imaging, Liaocheng Brain Hospital, 45 Huashan Road, Liaocheng, Shandong 252000, P.R. China

E-mail: dgwqf9@163.com

Key words: CT perfusion imaging, CT scans (non-contrast CT), acute ischemic stroke, thrombolytic therapy, application value worthy of being promoted in the clinical diagnosis and treatment of AIS patients.

\section{Introduction}

Cerebral stroke (CS) is an acute cerebrovascular disease, it is the main cause of disability in adults, it has a high incidence rate, high mortality rate and a high disability rate (1). The incidence rate of ischemic stroke in CS was higher than that of the hemorrhagic stroke (2). Acute ischemic stroke (AIS) is a circulatory disorder that seriously harms human health, and commonly occurs in middle-aged and elderly individuals (3). AIS is caused by atherosclerosis and thrombosis in patients, so it causes local brain tissue ischemia and hypoxic lesions in patients. The treatment process is extremely difficult (4). Currently, early application of recombinant tissue plasminogen activator (rt-PA) intravenous thrombolytic therapy can effectively improve the treatment of AIS (3). The key to the treatment of AIS is early intravenous administration of rt-PA intravenous thrombolytic therapy, which recanalizes the damaged blood vessels. Also the ischemic penumbra zone can be reperfused (5). Therefore, early diagnosis screening is critical for the treatment of AIS.

Guided by the American Heart Association/American Stroke Association Early Management Guide for AIS in 2018, all patients with suspected acute CA admitted to Liaocheng Brain Hospital (Liaocheng, China) should have a brain imaging assessment immediately after they arrive at Liaocheng Brain Hospital. In most cases, CT scans [non-contrast CT (NCCT)] can provide the necessary information for emergency assessment (6). A large number of documents have shown that the CT perfusion (CTP) has an important effect in the diagnosis of AIS $(7,8)$. CTP uses continuous dynamic scanning for selected levels of interest, with a non-ionic contrast agent to reflect cerebral blood flow (CBF), cerebral blood volume (CBV), mean transit time (MTT) and other blood perfusion parameters of related brain tissue via calculation, that has been recognized clinically (9). A large number of reports have shown that CTP related parameters can detect cerebral ischemic lesions at an early stage. This suggests that abnormal brain tissue can provide an effective basis for clinical diagnosis and the treatment of cerebrovascular diseases (10-12). In this research, we studied the clinical value of brain CTP in thrombolytic therapy of AIS patients and provided references for the treatment of AIS patients. 


\section{Patients and methods}

Basic patient information. A retrospective analysis of 185 patients diagnosed with AIS in Liaocheng Brain Hospital from April 2012 to December 2017 was carried out. The age range was 59 to 81 years, the average age was $71.15 \pm 5.22$ years, and the average time from pathogenesis to thrombolysis was $264.45 \pm 82.97 \mathrm{~min}$. Inclusion criteria were: i) pathogenesis duration $<12 \mathrm{~h}$; ii) ranked 4 to 24 points according to the National Institute of Health Stroke Scale (NIHSS) before treatment; iii) patients with effective treatment (2216) (NIHSS score decreased 4 or more points compared with that before treatment); iv) agree to receive relevant examinations and treatments; v) patients with history of complete cases and follow-up data; and vi) no related anticoagulation or thrombolytic therapy was received in other hospitals. Exclusion criteria were: i) patients who had allergic reactions or contraindications to the medicines of our research; ii) patients during pregnancy or lactation; iii) patients with acute gastrointestinal hemorrhage or other hemorrhage disorders; iv) patients with other serious diseases or tumors; v) patients with abnormal kidney, liver, coagulation, blood pressure and blood sugar; and vi) patients with communication or cognitive disorders. The subjects or their families signed informed consent and cooperated with the medical staff to complete the relevant medical treatment (Table I). The study was approved by the Ethics Committee of Liaocheng Brain Hospital.

NCCT, CTP inspection methods. Patients were requested to fast for 4-6 h before examination, paralyzed patients were asked to remove metal objects from the scanning range after the iodine allergy test became negative. The Toshiba Aquilion 64-slice spiral CT machine was used to scan the basal ganglia region for the level of interest. The parameters are as follows: scanning layer thickness was $5 \mathrm{~mm}$, matrix $512 \times 512$, tube voltage $120 \mathrm{kV}$, tube current $210 \mathrm{~mA}$, interval time: $1 \mathrm{sec}$; scanning time: $16 \mathrm{sec}$, a total of 80 layers and scanning range was $80 \mathrm{~mm}$. The non-ionic iodine contrast agent of $50 \mathrm{ml}$ of a venous injection $(370 \mathrm{mg} / \mathrm{ml})$ (iodoparin, cat. no. 60166-93-0; TargetMol, Boston, MA, USA) was injected through the cubital vein at a flow rate of 4.5 to $5.0 \mathrm{ml} / \mathrm{sec}$, and scanned at the same time. The CT image data was transmitted to the treatment workstation, and the treatment software of the system was used for analysis and a series of brain perfusion parameter maps were obtained. The abnormal area in the patient's CTP image was observed and the CTP parameters were measured, namely $\mathrm{CBF}, \mathrm{CBV}$, and MTT.

rt-PA thrombolytic therapy and drug schedule. The patients were connected to the monitor before treatment (Wuhan Kaijin Medical Technology Co., Ltd., Wuhan, China) and the changes in vital signs such as heart rate, blood pressure and breathing were closely examined. Also $0.9 \mathrm{mg} / \mathrm{kg}$ of alteplase (article no. RK20180329n; Boehringer Ingelheim Pharma GmbH $\&$ Co. KG, Ingelheim, Germany) were given within $4.5 \mathrm{~h}$ of pathogenesis for rt-PA thrombolytic therapy. The dosage was $0.9 \mathrm{mg} / \mathrm{kg}$, and the total dosage could not exceed $90 \mathrm{mg}$. Then, $10 \%$ of the total dose was injected intravenously within $1 \mathrm{~min}$, and the remaining drug was continuously infused intravenously for more than $1 \mathrm{~h}$. CT examination was performed $24 \mathrm{~h}$ after rt-PA thrombolysis and if hemorrhage did not occur in patients then routinely oral $100 \mathrm{mg}$ enteric-coated aspirin was applied, once per day.

Judging criteria. Early AIS signs of NCCT examination (13): blurred outline of the lenticular nucleus or reduced density levels; increased density of internal carotid artery in the brain (compact arterial sign); island gray-white interface disappears (island sign). The ischemic penumbra in CTP examination (14): CBF decreased significantly while CBV remained normal, mildly elevated or mildly decreased, and MTT was prolonged. The diagnosis of AIS patients with NCCT and CTP was observed, the CTP parameters in the abnormal perfusion zone and the mirror side zone of AIS patients were recorded and compared; the NIHSS score was used to investigate the correlation between the prognosis of NIHSS score and CTP parameters after 3 months of thrombolytic therapy.

Statistical analysis. Statistical analysis was conducted by SPSS 17.3 (Beijing Net Counting Times Technology Co., Ltd., Beijing, China) software system. Basic patient data counting were expressed as percentage (\%), using a Chi-square test. The CTP parameters of CBV, CBF, and MTT were expressed as mean \pm standard deviation and the difference between the groups was analyzed by t-test. The correlation between NIHSS scores and CTP parameters of CBV, CBF, and MTT was analyzed by Spearman correlation analysis after 3 months of thrombolytic therapy. $\mathrm{P}<0.05$ was considered to indicate a statistically significant difference.

\section{Results}

Comparison between CTP and CTA examinations in the diagnosis of AIS patients. In total, 177 patients were diagnosed with AIS by clinical manifestations, biochemical tests and imaging. The sensitivity level of CIS examination for diagnosis of AIS patients was $96.61 \%$ and the sensitivity level of NCCT for diagnosis of AIS patients was $63.28 \%$. The sensitivity level of CTP examination for diagnosis of AIS patients was significantly higher than that of the NCCT examination and the difference was statistically significant $(\mathrm{P}<0.050)$. The specificity level of CIS examination for diagnosis of AIS patients was $50.00 \%$ and the specificity level of NCCT for diagnosis of AIS patients was $25.00 \%$. The specificity level of patients with AIS diagnosed by CTP was significantly higher than that of the NCCT, the difference was statistically significant $(\mathrm{P}<0.050)$. The diagnostic compliance rate of patients with AIS diagnosed by CTP was $94.59 \%$ and the diagnostic compliance rate of AIS diagnosed by CT scan was $61.62 \%$. The diagnostic compliance rate of patients with AIS diagnosed by CTP was significantly higher than that of NCCT and the difference was statistically significant $(\mathrm{P}<0.050$; Tables II and III and Fig. 1).

Changes in $C B V$ of CTP parameters and correlation with NIHSS scores. The CBV in the abnormal perfusion zone of AIS patients was $4.26 \pm 0.61 \mathrm{ml} / 100 \mathrm{~g}$ and in the mirror side zone was $5.95 \pm 0.84 \mathrm{ml} / 100 \mathrm{~g}$. The CBV in the abnormal perfusion zone was significantly lower than that of the mirror side zone, and the difference was statistically significant $(t=21.160, P<0.001)$. NIHSS scores were obtained 3 months 
Table I. General information of patients in clinics $(n=185)$.

\begin{tabular}{lr}
\hline Factors & $\mathrm{n}(\%)$ \\
\hline Age (years) & \\
$<70$ & $73(39.46)$ \\
$\geq 70$ & $112(60.54)$ \\
Sex & \\
Male & $124(67.03)$ \\
Female & $61(32.97)$ \\
Married or single & \\
Single & $62(33.51)$ \\
Married & $123(66.49)$ \\
History of smoking & \\
Smoking & $136(73.51)$ \\
Non-smoking & $49(26.49)$ \\
History of drinking alcohol & \\
Alcoholic & $118(63.78)$ \\
Non-alcoholic & $67(36.22)$ \\
Eating habits & \\
Greasy & $147(79.46)$ \\
Light & $38(20.54)$ \\
Type & \\
Carotid artery & \\
Vertebral artery & \\
\hline
\end{tabular}

Table II. AIS effectiveness in CTP diagnosis.

\begin{tabular}{lccc}
\hline CTP diagnosis & $\begin{array}{c}\text { Clinical } \\
\text { diagnosis (+) }\end{array}$ & $\begin{array}{c}\text { Clinical } \\
\text { diagnosis (-) }\end{array}$ & Total \\
\hline CTP diagnosis (+) & 171 & 4 & 175 \\
CTP diagnosis (-) & 6 & 4 & 10 \\
Total & 177 & 8 & 185 \\
\hline
\end{tabular}

AIS, acute ischemic stroke; CTP, CT perfusion.

after thrombolytic therapy and showed that the prognostic NIHSS score was negatively correlated with CBV in patients with thrombolytic therapy ( $\mathrm{r}=-0.912, \mathrm{P}<0.001$; Fig. 2).

Changes in CBF of CTP parameters and their correlation with NIHSS scores. The CBF in the abnormal perfusion zone of AIS patients was $45.58 \pm 6.07 \mathrm{ml} / 100 \mathrm{~g} / \mathrm{min}$ and in the mirror side zone was $59.41 \pm 7.38 \mathrm{ml} / 100 \mathrm{~g} / \mathrm{min}$. The difference was statistically significant $(\mathrm{t}=19.170, \mathrm{P}<0.001)$. NIHSS scores were obtained 3 months after thrombolytic therapy and showed that the prognostic NIHSS score was negatively correlated with $\mathrm{CBF}$ in patients with thrombolytic therapy $(\mathrm{r}=-0.915, \mathrm{P}<0.001$; Fig. 3).

Changes in MTT of CTP parameters and their correlation with NIHSS scores. The MTT in the abnormal perfusion zone of AIS patients was $4.96 \pm 0.72 \mathrm{sec}$ and the MTT in the
Table III. AIS effectiveness in NCCT diagnosis.

\begin{tabular}{lccc}
\hline & $\begin{array}{c}\text { Clinical } \\
\text { diagnosis } \\
\text { NCCT diagnosis }\end{array}$ & $\begin{array}{c}\text { Clinical diagnosis } \\
\text { (Mild brain } \\
\text { injury) }\end{array}$ & Total \\
\hline NCCT diagnosis (+) & 112 & 6 & 118 \\
NCCT diagnosis (-) & 65 & 2 & 67 \\
Total & 177 & 8 & 185 \\
\hline
\end{tabular}

AIS, acute ischemic stroke; NCCT, non-contrast CT.

mirror side zone was $4.02 \pm 0.56 \mathrm{sec}$. The MTT in the abnormal perfusion zone was higher than that of the mirror side zone and the difference was statistically significant $(\mathrm{t}=13.480, \mathrm{P}<0.001)$. NIHSS scores were obtained 3 months after thrombolytic therapy and showed that the prognostic NIHSS scores were positively correlated with MTT in patients with thrombolytic therapy ( $r=0.887, \mathrm{P}<0.001$; Fig. 4).

\section{Discussion}

According to statistics, in industrialized countries, cerebrovascular disease is the leading cause of death for women. Also it is the second cause of death for men and is an important cause of cognitive impairment and dementia $(15,16)$. CA is the main cause of functional disability, and most CA patients have neurological sequelae after treatment and it becomes impossible to restore the same level of daily living activities as before the disease (17). Currently, the number of patients with global AIS is gradually declining due to early control of risk factors. However, many patients still have to take long-term care after treatment, causing a huge burden on the patient's family and society. Therefore, early treatment is very important (18). The first choice for early treatment of AIS is rt-PA intravenous thrombolytic therapy, and rt-PA is a highly selective thrombolytic drug for fibrin (19). rt-PA is activated once it binds to fibrin, this will induce the plasminogen convert to fibrinolytic enzyme, which leads to the degradation of fibrin and thrombosis in the body (20). rt-PA thrombolytic therapy can restore blood supply to the originally blocked blood vessels and reperfusion of brain tissue in the ischemic penumbra. This improves the prognosis for life quality of patients (21). This study involved 185 patients diagnosed with AIS in Liaocheng Brain Hospital, and the consistency of NCCT and CTP examination and clinical diagnosis in AIS patients were analyzed. The correlation of the prognosis of NIHSS scores between AIS patients and CBV, CBF, MTT of CTP parameters were also analyzed. This study provided references for clinical diagnosis and treatment of AIS patients.

In this study, 177 patients were diagnosed with AIS through clinical manifestations, biochemical tests and imaging. Upon comparison of the detection results between the CTP and NCCT of imaging, we revealed that the sensitivity, specificity, and diagnostic compliance rates of AIS patients diagnosed by CTP were higher than those of NCCT and the difference was statistically significant. With the continuous development and progress of modern medical CT scanning technology, CT has 


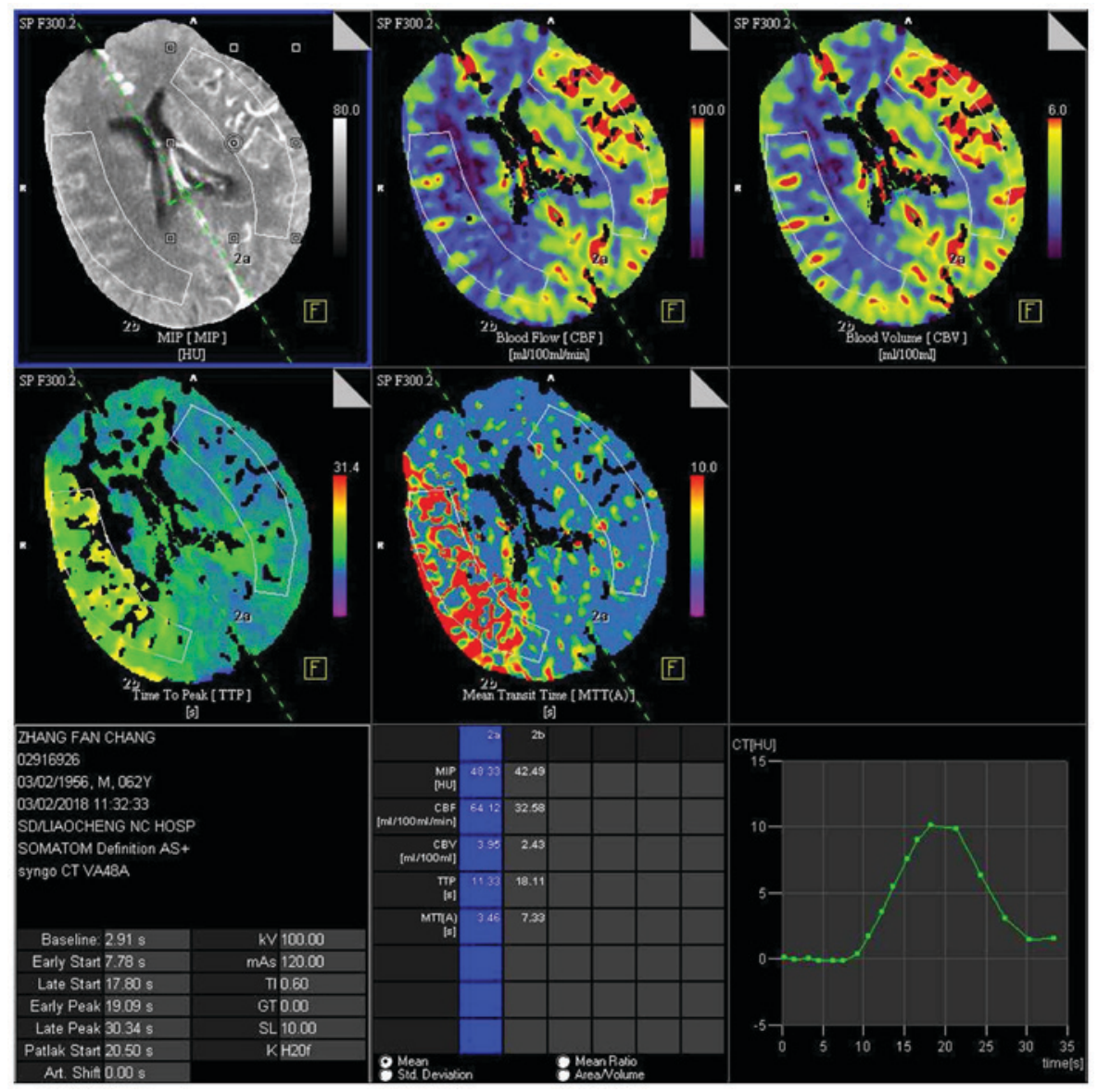

Figure 1. CTP image of AIS patients. MTT prolonged in the ischemic penumbra, CBF and CBV decreased. CTP, CT perfusion; AIS, acute ischemic stroke; MTT, mean transit time; CBF, cerebral blood flow; CBV, cerebral blood volume.

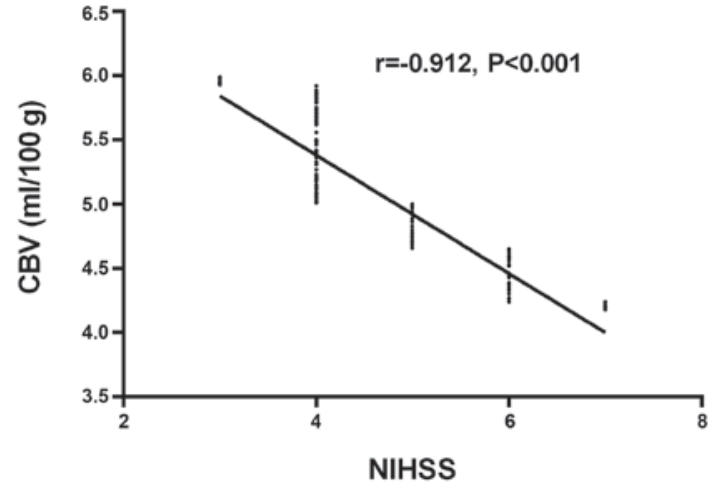

Figure 2. Correlation between CBV of CTP parameters and NIHSS scores. NIHSS scores were obtained 3 months after thrombolytic therapy, and the prognostic NIHSS score was negatively correlated with CBV $(\mathrm{r}=-0.912$ $\mathrm{P}<0.001)$. CBV, cerebral blood volume; CTP, CT perfusion; NIHSS, National Institute of Health Stroke Scale.

developed from morphological imaging diagnosis to assessable hemodynamic changes that can be assessed in vivo tissue (22). According to Yoo et al (23), it was found that the safety and efficacy of early NCCT for intra-arterial treatment has a diagnostic value only for patients with small infarction, while patients with large infraction require further examination for diagnosis. However, Finlayson et al (24) showed that the diagnostic value of CTP was higher than that of the NCCT and angiography in the diagnosis of acute CA patients using NCCT, CT angiography and CTP,

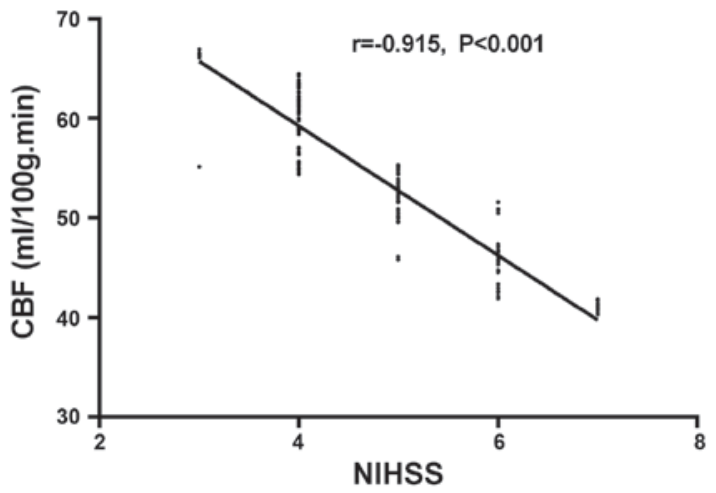

Figure 3. Correlation between CBF of CTP parameters and NIHSS score. NIHSS scores were obtained 3 months after thrombolytic therapy and the prognosis of patients with thrombolytic therapy was negatively correlated with $\mathrm{CBF}(\mathrm{r}=-0.915, \mathrm{P}<0.001)$. CBF, cerebral blood flow; CTP, CT perfusion; NIHSS, National Institute of Health Stroke Scale.

which further approved our point of view. Later, we studied the $\mathrm{CBV}, \mathrm{CBF}$, and MTT between the abnormal perfusion zone of AIS patients and the mirror side zone. It was found that the CBV and $\mathrm{CBF}$ in the abnormal perfusion zone were significantly lower than that of the mirror side zone. Also the MTT in the abnormal perfusion zone was significantly lower than that of the mirror side zone and the difference was statistically significant. Relevant parameters of CTP can reflect the collateral circulation of patients and the abnormal perfusion of hemodynamics in 


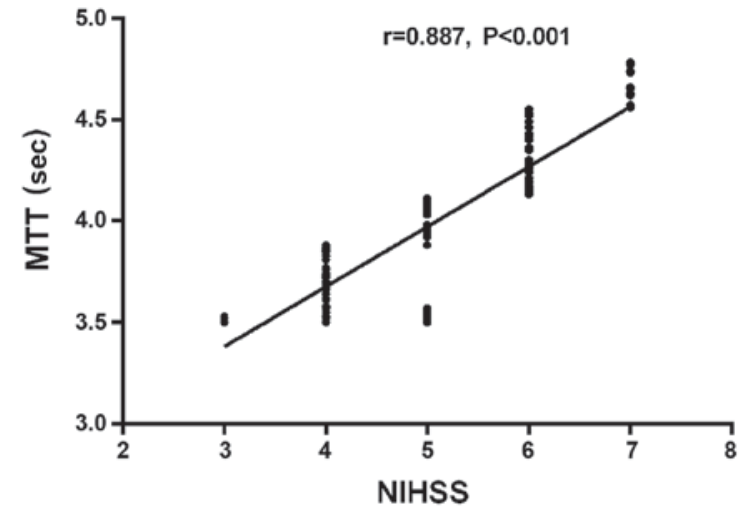

Figure 4. Correlation between MTT of CTP parameters and NIHSS score. NIHSS scores were obtained 3 months after thrombolytic therapy and the prognostic NIHSS scores were positively correlated with MTT ( $\mathrm{r}=0.887$, $\mathrm{P}<0.001)$. MTT, mean transit time; CTP, CT perfusion; NIHSS, National Institute of Health Stroke Scale.

brain tissues, so it provides a basis for clinical treatment. If the $\mathrm{CBF}$ declines slightly, it indicates that the cerebral circulation reserve is decompensated. Also a significant reduction indicates that the patient may have developed a cerebral infarction. Delayed MTT suggests a deduced cerebral perfusion pressure as well as impaired perfusion reserve and can indicate the condition of the patient's collateral circulation (25). However, there is no consistent conclusion on the optimal thresholds for CBV, CBF, and MTT between the abnormal perfusion zone and the mirror side zone. Therefore, we studied whether the prognosis of patients after treatment was related to the pre-treatment CTP parameters. NIHSS scores were obtained 3 months after thrombolytic therapy and the prognosis of patients with thrombolytic therapy was negatively correlated with $\mathrm{CBV}$ and $\mathrm{CBF}$, while it was positively correlated with MTT, and the difference was statistically significant. According to van Seeters et al (26) in a study of patients with suspected AIS, the parameters of CTP examination at admission have a strong predictive effect on patients with poor prognosis and can be used to predict long-term clinical outcomes. However, Ma et al (27) also found that CTP parameters are correlated with the evaluation index of patients' clinical prognosis on the 14th and 90th day, which further supports our research results.

In this experiment, due to the small number of patients with AIS in Liaocheng Brain Hospital, we only have a small base of selected subjects. Therefore, there may exist contingency within our results and there were a large number of research variables in AIS patients. Further study is still required.

Overall, brain CTP has a high diagnostic value for rt-PA intravenous thrombolytic therapy in AIS. Also there is a significant correlation with patients' prognosis score, which is worthy of being promoted in the clinical diagnosis and treatment of AIS patients.

\section{Acknowledgements}

Not applicable.

\section{Funding}

No funding was received.

\section{Availability of data and materials}

The datasets used and/or analyzed during the present study are available from the corresponding author on reasonable request.

\section{Authors' contributions}

SX wrote the manuscript. SX and LZ recorded and analyzed NCCT and CTP inspection results. LW and LZ were responsible for rt-PA thrombolytic therapy. All authors read and approved the final manuscript.

\section{Ethics approval and consent to participate}

The study was approved by the Ethics Committee of Liaocheng Brain Hospital (Liaocheng, China). Signed informed consents were obtained from the patients or guardians.

\section{Patient consent for publication}

Not applicable.

\section{Competing interests}

The authors declare that they have no competing interests.

\section{References}

1. Sun Y, Zhang G, Zhang Z, Yu P, Zhong H, Du J and Wang Y: Novel multi-functional nitrones for treatment of ischemic stroke. Bioorg Med Chem 20: 3939-3945, 2012.

2. Amarenco P, Lavallée PC, Labreuche J, Albers GW, Bornstein NM, Canhão P, Caplan LR, Donnan GA, Ferro JM, Hennerici MG, et al; TIÁregistry.org Investigators: One-year risk of stroke after transient ischemic attack or minor stroke. N Engl J Med 374: 1533-1542, 2016.

3. Jauch EC, Saver JL, Adams HP Jr, Bruno A, Connors JJ, Demaerschalk BM, Khatri P, McMullan PW Jr, Qureshi AI, Rosenfield K, et al; American Heart Association Stroke Council; Council on Cardiovascular Nursing; Council on Peripheral Vascular Disease; Council on Clinical Cardiology: Guidelines for the early management of patients with acute ischemic stroke: a guideline for healthcare professionals from the American Heart Association/American Stroke Association. Stroke 44: 870-947, 2013.

4. Minnerup J, Wersching H, Teuber A, Wellmann J, Eyding J, Weber R, Reimann G, Weber W, Krause LU, Kurth T, et al; REVASK Investigators: Outcome after thrombectomy and intravenous thrombolysis in patients with acute ischemic stroke: a prospective observational study. Stroke 47: 1584-1592, 2016.

5. Joux J, Olindo S, Girard-Claudon A, Chausson N, Saint-Vil M, Signate A, Edimonana M, Jeannin S, Aveillan M, Cabre P, et al: Prehospital transfer medicalization increases thrombolysis rate in acute ischemic stroke. A French stroke unit experience. Clin Neurol Neurosurg 115: 1583-1585, 2013.

6. Powers WJ, Rabinstein AA, Ackerson T, Adeoye OM, Bambakidis NC, Becker K, Biller J, Brown M, Demaerschalk BM, Hoh B, et al; American Heart Association Stroke Council: 2018 guidelines for the early management of patients with acute ischemic stroke: a guideline for healthcare professionals from the American Heart Association/American Stroke Association. Stroke 49: e46-e110, 2018.

7. Wintermark M: Brain perfusion-CT in acute stroke patients. Eur Radiol 15 (Suppl 4): D28-D31, 2005.

8. Lövblad KO and Baird AE: Computed tomography in acute ischemic stroke. Neuroradiology 52: 175-187, 2010.

9. Wang XC, Gao PY, Xue J, Liu GR and Ma L: Identification of infarct core and penumbra in acute stroke using CT perfusion source images. AJNR Am J Neuroradiol 31: 34-39, 2010. 
10. Borst J, Berkhemer OA, Roos YB, van Bavel E, van Zwam WH, van Oostenbrugge RJ, van Walderveen MA, Lingsma HF, van der Lugt A, Dippel DW, et al; MR CLEAN investigators: value of computed tomographic perfusion-based patient selection for intra-arterial acute ischemic stroke treatment. Stroke 46 : 3375-3382, 2015

11. Vagal A, Menon BK, Foster LD, Livorine A, Yeatts SD, Qazi E, d'Esterre C, Shi J, Demchuk AM, Hill MD, et al: Association between $\mathrm{CT}$ angiogram collaterals and $\mathrm{CT}$ perfusion in the interventional management of stroke III trial. Stroke 47: 535-538, 2016.

12. Austein F, Riedel C, Kerby T, Meyne J, Binder A, Lindner T, Huhndorf M, Wodarg F and Jansen O: Comparison of perfusion CT software to predict the final infarct volume after thrombectomy. Stroke 47: 2311-2317, 2016.

13. Demchuk AM, Menon BK and Goyal M: Comparing vessel imaging: noncontrast computed tomography/computed tomographic angiography should be the new minimum standard in acute disabling Stroke. Stroke 47: 273-281, 2016.

14. Flottmann F, Broocks G, Faizy TD, Ernst M, Forkert ND, Grosser M, Thomalla G, Siemonsen S, Fiehler J and Kemmling A: CT-perfusion stroke imaging: a threshold free probabilistic approach to predict infarct volume compared to traditional ischemic thresholds. Sci Rep 7: 6679, 2017.

15. Guidelines for diagnosis and management of cardiovascular sequelae in Kawasaki disease (JCS 2003). J Cardiol 43: 263-283, 2004 (In Japanese).

16. Kalaria RN: Cerebrovascular disease and mechanisms of cognitive impairment: evidence from clinicopathological studies in humans. Stroke 43: 2526-2534, 2012.

17. Allen CL and Bayraktutan U: Risk factors for ischaemic stroke. Int J Stroke 3: 105-116, 2008.

18. Bergström L, Irewall AL, Söderström L, Ögren J, Laurell K and Mooe T: One-year incidence, time trends, and predictors of recurrent ischemic stroke in Sweden from 1998 to 2010: an Observational Study. Stroke 48: 2046-2051, 2017.

19. Ntaios G, Dziedzic T, Michel P, Papavasileiou V, Petersson J, Staykov D, Thomas B and Steiner T; European Stroke Organisation: European Stroke Organisation (ESO) guidelines for the management of temperature in patients with acute ischemic stroke. Int J Stroke 10: 941-949, 2015.
20. Arba F, Inzitari D, Ali M, Warach SJ, Luby M and Lees KR; STIR/VISTA Imaging Collaboration: Small vessel disease and clinical outcomes after IV rt-PA treatment. Acta Neurol Scand 136: 72-77, 2017

21. Akutagawa N, Sadashima S, Nakagaki H, Nagano S and Yoshimura T: Intracerebral hemorrhage after intravenous recombinant tissue plasminogen activator (rt-PA) therapy for acute cerebral infarction in a patient with ANCA-associated vasculitis. Rinsho Shinkeigaku 57: 454-456, 2017.

22. Wang J, Wu N, Cham MD and Song Y: Tumor response in patients with advanced non-small cell lung cancer: Perfusion CT evaluation of chemotherapy and radiation therapy. AJR Am J Roentgenol 193: 1090-1096, 2009.

23. Yoo AJ, Berkhemer OA, Fransen PSS, van den Berg LA, Beumer D, Lingsma HF, Schonewille WJ, Sprengers MES van den Berg R, van Walderveen MAA, et al; MR CLEAN investigators: effect of baseline Alberta Stroke Program Early CT Score on safety and efficacy of intra-arterial treatment: a subgroup analysis of a randomised phase 3 trial (MR CLEAN). Lancet Neurol 15: 685-694, 2016.

24. Finlayson O, John V, Yeung R, Dowlatshahi D, Howard P, Zhang L, Swartz R and Aviv RI: Interobserver agreement of ASPECT score distribution for noncontrast CT, CT angiography, and CT perfusion in acute stroke. Stroke 44: 234-236, 2013.

25. Yu Y, Han Q, Ding X, Chen Q, Ye K, Zhang S, Yan S, Campbell BC, Parsons MW, Wang S, et al: Defining core and penumbra in ischemic stroke: A voxel- and volume-based analysis of whole brain CT perfusion. Sci Rep 6: 20932, 2016.

26. van Seeters T, Biessels GJ, Kappelle LJ, van der Schaaf IC, Dankbaar JW, Horsch AD, Niesten JM, Luitse MJ, Majoie CB, Vos JA, et al; Dutch acute stroke study (DUST) investigators: The prognostic value of CT angiography and CT perfusion in acute ischemic stroke. Cerebrovasc Dis 40: 258-269, 2015.

27. Ma QF, Jia JP, Wu J, Xu EH, Yu YY, Lu J and Zhang M: Relationship between computed tomography perfusion imaging and prognosis in hyperacute cerebral infarction. Zhonghua Yi Xue Za Zhi 91: 3337-3340, 2011 (In Chinese).

This work is licensed under a Creative Commons Attribution-NonCommercial-NoDerivatives 4.0 International (CC BY-NC-ND 4.0) License. 